\title{
An analogy to the Reynolds number for the neutral gas component of a weak plasma
}

\author{
C. M. Hall \\ Troms $\varnothing$ Geophysical Observatory, Faculty of Science, University of Troms $\varnothing, 9037$ Troms $\varnothing$, Norway
}

Received: 5 June 2000 - Revised: 6 October 2000 - Accepted: 6 October 2000

\begin{abstract}
The Reynolds number $R e$ is used as a metric to assess whether or not a flow may contain turbulence. In a weakly ionised gas with an external electric field imposed, ions exert a drag on the neutral particles. Thus, a component of the neutral motion is attributable to the ion-drag. An analogy to $R e$ has been proposed in which the ion-drag-induced velocity contribution to the neutral motion is used. This analogy thus represents the destabilising effect of the electric field on the neutral dynamics. Here quantisation of this proposed metric is investigated.
\end{abstract}

Key words. Meteorology and atmospheric dynamics (turbulence) - Ionosphere (ionosphere-atmosphere interactions; electric fields and currents)

\section{Introduction}

Various reports, e.g. Danilov et al. (1979), exist which indicate that neutral air turbulence in the lower thermosphere may be enhanced during periods of increased geomagnetic activity. Fluctuations in the Earth's magnetic field are invariably associated with current systems in the upper atmosphere and hence with electric fields. Hall (2000) attempted to explain the observations of, in particular, Danilov et al. (1979), by investigating how realistic neutral wind profiles in the lower thermosphere exhibited shears giving bulk Richardson numbers $(R i)$ indicative of dynamic instability. The Reynolds number $(R e)$ is also used to identify situations in which flows may become (or cease to become) turbulent. While the usual formulation of $R e$ involves the bulk speed of the fluid and a typical length scale, the turbulent Reynolds number uses eddy velocity and size. To illustrate this, an initially laminar flow may become turbulent as a result of increasing flow speed when a certain critical $R e$ is reached. Only after turbulence has been established may the turbulent $R e$ be identified; subsequently, as the flow subsides for example, the turbulence will cease at a certain value of $R e$ which need not be

Correspondence to: C. Hall (chris.hall@ phys.uit.no) the same as for turbulence instigation. In other words, different kinds of $R e$ may be defined, and furthermore they often exhibit a hysteresis. While an $R e$ for laminar flow may exceed several hundred (dependent on geometry) before turbulence sets in, the condition of turbulent $R e<1$ indicates that viscosity dominates to such a degree that turbulent eddies cannot develop. Thus, identifying the contribution collisions from ions make to the neutral velocity can be used to construct another analogy to the classic Reynolds number. When this number is less than some critical value, viscosity dominates the dynamics such that turbulence is suppressed. Combining profiles of $R i$ with this turbulence-possible/suppressed metric would allow one to identify regions in the atmosphere at which electrodynamics is capable of destabilising the neutral flow, or at least augmenting existing turbulence. High in the lower thermosphere the neutral viscosity (which increases exponentially with height) is so great that neutral air turbulence is precluded. Lower down, in the mesosphere, although neutral air turbulence may well be present, the ionisation is so little that the ions are unable to influence the neutral dynamics to any degree. Hall (2000) found that in between these regions, it was feasible for the electrodynamics to induce or enhance neutral turbulence. It is at these intermediate heights that such an effect could explain the findings of Danilov et al. (1979).

Hall (2000) discussed some possible formulations of the $R e$ analogy number, which will be denoted $\aleph$ here. In this treatise, the quantisation of $\aleph$ will be investigated in more detail in an attempt to arrive at a formulation that may be experimentally tested in subsequent work.

\section{The ion-drag analogy to $R e$}

The Reynolds number $R e$ is usually expressed as:

$R e=\frac{U L}{v}$

where $U$ is a typical velocity, $L$ is a typical length and $v$ is the kinematic viscosity. As an example, consider flow in a pipe: 
Reynolds' classic experiment measured the average velocity by measuring the volume of fluid which passed through the pipe in a given time interval. $L$ was the internal diameter of the pipe (obviously, a transverse length); a critical number of 2000 resulted from his pipe flow experiments. In the boundary layer, the boundary layer thickness is often used as $L$ (again, transverse) while in applications to jet flow in a medium, a longitudinal length is sometimes used. In the free atmosphere the specification of a bulk $L$ is difficult if not impossible and, almost no matter how they are calculated, bulk Reynolds numbers are usually so large that their value rarely precludes turbulence. In such environments, $R i$ is more often used as the turbulence metric. An alternative to this approach is to consider time scales, therefore. The Peclet number is defined as the ratio of molecular, $T_{m}$, to turbulent, $T_{t}$, time scales. From, e.g. Tennekes and Lumley (1972):

$T_{t}=L / u$

where $u$ is a turbulent velocity scale, and

$T_{m}=L^{2} / \gamma$

where $\gamma$ is the thermal conductivity (we can see this is dimensionally correct since thermal conductivity and diffusivity have dimensions $L^{2} T^{-1}$ ),

$\frac{T_{t}}{T_{m}} \approx \frac{\gamma}{u L}$

The ratio $v / \gamma$ is the molecular Prandtl number, which for air $=0.73$, but taking it as unity for our purposes we see that

$\frac{T_{t}}{T_{m}} \approx \frac{1}{R e}$.

In this argument lies the intrinsic assumption that the same length scale can be used for both processes, and this is not usually true at high $R e$.

In the atmosphere, there exists a height (or possibly heights), the turbopause, at which turbulent and molecular diffusivities are equal. From Eq. (5) we can see that this is the height at which turbulent $R e$ is unity. The Hall (2000) analogy to $R e$ which rather than representing the ratio of inertial (or turbulent) effects to viscosity (or molecular effects), represents the ratio of electrodynamically induced inertia to viscosity. Similarly to $R e$, when viscosity is sufficiently large, its damping inhibits inertial effects induced by electrodynamics. Hall (2000) utilised Eq. (5) thus:

$T_{m}=L_{B}^{2} / v$

$T_{\text {electrodynamic }}=1 / \nu_{\mathrm{ni}}$

where $L_{B}$ is the buoyancy length scale, $T_{\text {electrodynamic }}$ is a time scale for electrodynamics induced inertia, and $v_{\mathrm{ni}}$ is the neutral-ion collision frequency. The quantity $L_{B}$ may be thought of as the largest size of an eddy (if such a structure indeed exists). The reciprocal of the neutral-ion collision frequency represents the mean time between successive collisions of a given neutral molecule with any ion. This does not represent the time scale for acceleration of the neutrals due to an ion motion induced by an external electric field. While the proposal:

$\aleph \equiv \frac{\text { (ion-drag induced neutral wind) } \times \text { length }}{\text { kinematic viscosity }}$

and suggested implementation:

$\aleph=\frac{T_{m}}{T_{\text {electrodynamic }}}$

are both valid, the Hall (2000) derivation of $T_{\text {electrodynamic }}$ needs improvement. We shall return, therefore, to formulation (8).

Consider a collisional plasma in which the neutral particles are initially at rest and the ions move with a speed $v_{p}$. After a time $t$, the neutral particles will have attained a speed

$v_{a}=v_{p}\left[1-\exp \left(-v_{\mathrm{ni}} t\right)\right]$

(e.g. Ratcliffe, 1972, Eq. 6.39). Now for the numerator in Eq. (8) we may substitute (ion-drag induced neutral wind) ${ }^{2} \times$ time scale, and if we utilise the Brunt-Väisälä period $t_{B}$ as a typical time scale we have:

$\aleph=\frac{\left[v_{p}\left\{1-\exp \left(-v_{\mathrm{ni}} t_{B}\right)\right\}\right]^{2} t_{B}}{v}$

wherein we may obtain $v_{\text {ni }}$ from

$v_{\mathrm{ni}}=2.6 \cdot 10^{-15} n_{i} / M_{i}$

where $n_{i}$ is the ion number density and $M_{i}$ is the ion molecular weight (e.g. Ratcliffe, 1972).

While a Reynolds number analogy has been used in formulating $\aleph$, because of the very large value of the damping force, the kinematic viscosity, and the use of the BruntVäisälä period as the characteristic time constant of the destabilisation together with the ion drag velocity, in proposing a critical value for the transition from laminar to turbulent flow, a Richardson type criterion may be more appropriate, with a critical value of order 1 . While this value requires experimental verification, the assumption of a critical value near unity is consistent with the concept of geomagnetic influence on turbopause height, as shown in the following examples. Let us then test this formulation by substituting some realistic values. For the lower thermosphere, we shall use a mean molecular weight of $30.5 \mathrm{amu}$. Ion density profiles may be obtained from the international reference ionosphere (IRI) (Bilitza, 1990). The MSIS-E model atmosphere (Hedin, 1991) can be used to obtain a typical value for the Brunt-Väisälä period of $300 \mathrm{~s}$, and also for the kinematic viscosity. Fujii et al. (1998) provides a source of realistic ion wind speeds derived from incoherent scatter observations from a series of electric field $>25 \mathrm{mV} \mathrm{m}^{-1}$ cases. Figure 1 shows the resulting height profile of the proposed analogy to $R e$. Wherever the value falls to below unity, the viscous damping is more important than ion-drag effects. A maximum can be seen at $115 \mathrm{~km}$ altitude and what is of particular 


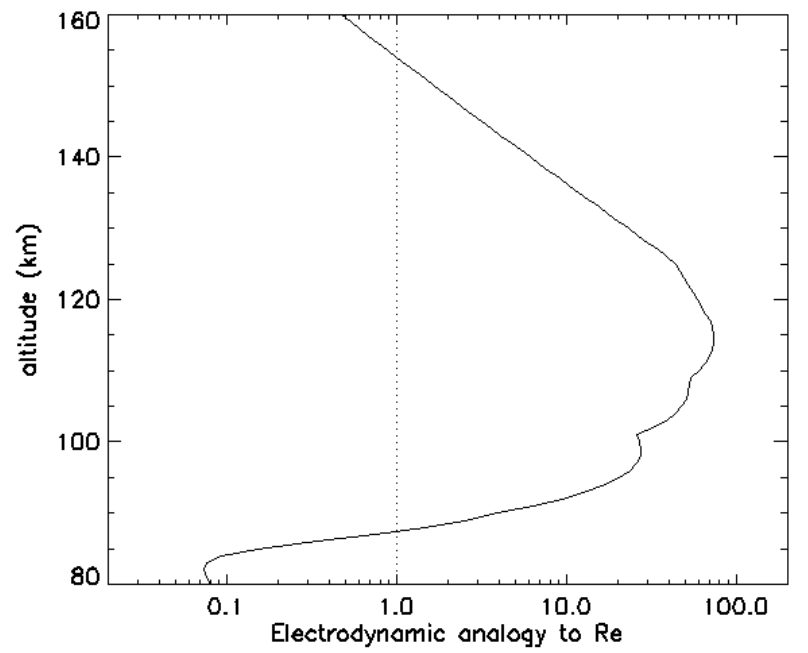

Fig. 1. The proposed (Hall, 2000) analogy to the Reynolds number for ion-drag induced neutral motion as a function of altitude and using a typical neutral wind speed profile from Fujii et al. (1998). The vertical dotted line indicates the unity condition.

interest here is that the turbopause normally occurs somewhere near this height. Depending on the critical value(s) for $\aleph$, sufficient ion-drag may well enhance or even induce turbulence causing the viscous cut-off to occur higher up than if no ions were present.

In the preceding example, we had used measured wind statistical values associated with an unknown electric field, and a nominal Brunt-Väisälä period (since Fujii et al., 1990, used data from many dates). Let us now take the electric field as the starting point and use the MSIS-E temperature profile to better estimate the Brunt-Väisälä period. The ion speed $v_{p}$ in the lower thermosphere may be expressed by

$v_{p}=\frac{e E}{v_{\text {in }} m_{i}}$

where $e$ is the electronic charge, $E$ is the strength of the external electric field, $v_{\text {in }}$ is the ion-neutral collision frequency and $m_{i}$ is the ion mass. Since the models used are timedependent, it has been possible to examine both summer and winter cases. The Re analogy is shown for both summer (Fig. 2a) and winter (Fig. 2b) and for three different external electric field strengths. The greatest electric field, $60 \mathrm{mV} \mathrm{m}^{-1}$ represents disturbed conditions. Even greater values might occur in reality but these would probably be shorter lived than the response time of the neutral gas. It can be seen that a well-defined region around the nominal turbopause height occurs, but only in the summer, and for field strengths of around $25 \mathrm{mV} \mathrm{m}^{-1}$ and greater, does the $R e$ analogy indicate dominance of electrodynamic driving over neutral viscosity. Figure 3 shows a 3-dimensional representation for a range of electric fields for the summer case (in winter the $R e$ analogy is always small). The shading in the figure indicates the region where the $R e$ analogy exceeds unity and it can be seen that the maximum lies around $105 \mathrm{~km}$ altitude.
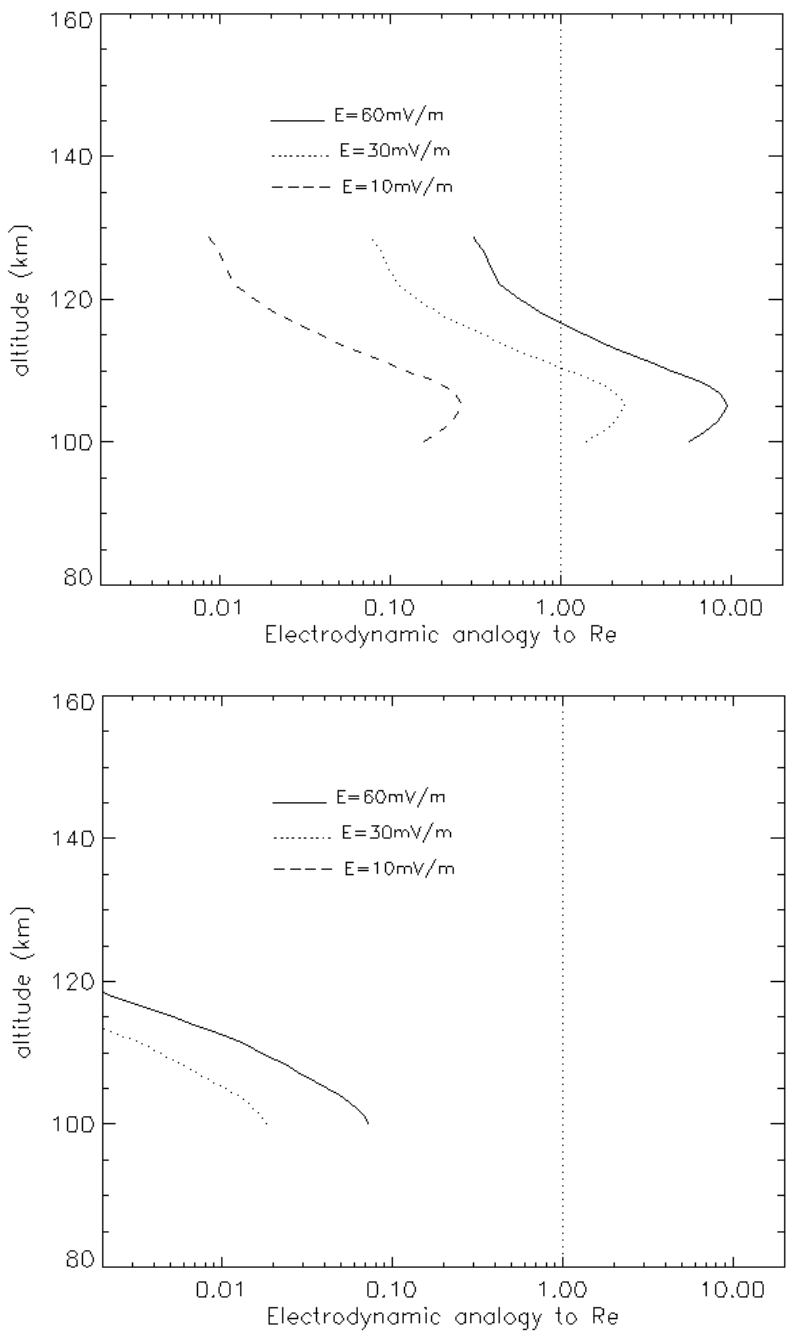

Fig. 2. The proposed analogy to the Reynolds number as a function of height. Different electric field strengths are used to derive corresponding plasma velocity profiles. (a) Summer case and (b) winter case. The vertical dotted lines indicate the unity condition.

\section{Conclusion}

An analogy to the Reynolds number has been proposed, in which the component of the neutral atmosphere inertia as induced by ion drag is compared with its kinematic viscosity. Often, turbulence is associated with high Reynolds numbers, but in this case it is more appropriate to consider the inverse: that sub-unity turbulent Reynolds numbers represent cases in which the kinematic viscosity is sufficient to damp out inertia effects before turbulent eddies have had chance to form. The ion-drag analogy to the Reynolds number should also be used in this way, although the critical value below which ion-drag driven neutral air turbulence is precluded has yet to be determined experimentally. The number is intended to represent only the influence by the ion drag on the neutral dynamics, and it should be remembered that, especially where the kinematic viscosity is small, other mechanisms come into play to determine whether turbulence can survive. Neither should 


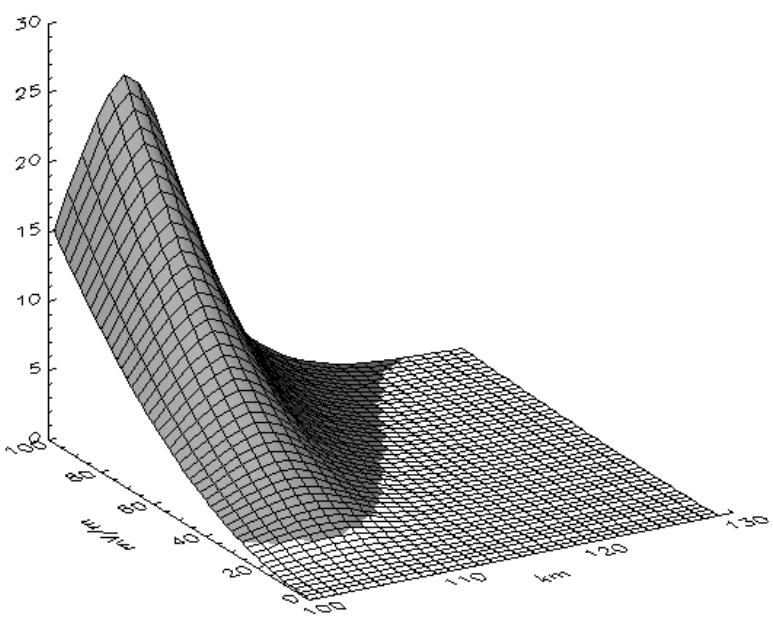

Fig. 3. The proposed analogy to the Reynolds number as a function of height and electric field strength, for summer conditions. The shaded area indicates where the metric exceeds unity.

the ion-drag Reynolds number analogy alone be used as an indicator of turbulence. If shears are present in the flow that exhibit Richardson numbers of $<0.25$, the Reynolds number analogy (and indeed the Reynolds number itself) determines whether the viscous damping is unimportant enough to allow turbulence to form. It would appear that ion drag is most efficient at inducing neutral air speeds sufficient that inertial effects overcome those of viscous damping, in an atmospheric layer peaking around $105 \mathrm{~km}$ altitude. Below this peak, the effect decreases with lessening altitude because ion concentrations decrease. Above the peak, the effect decreases with altitude due to the increasing kinematic viscosity. However, the height regime in which the ion-drag effects are significant coincides with the normal turbopause height, and therefore these effects may indeed be responsible for inducing or enhancing turbulence and therefore explaining the observed (Danilov et al., 1979) increases in turbopause height during periods of high geomagnetic activity.

Acknowledgement. Topical Editor Donal Murtagh thanks R. Roper and T. A. Blix for their help in evaluating this paper.

\section{References}

Bilitza, D. (Ed.), International Reference Ionosphere 1990, NSSDC 90-22, Greenbelt, Maryland, 1990.

Danilov, A. D., Kalgin, U. A., and Pokhunov, A. A., Variation of the mesopause level in polar regions, Space Res. XIX, 83, 173-176, 1979.

Fujii, R., Nozawa, S., Buchert, S. C., Matuura, N., and Brekke, A., The motion of ions in the auroral ionosphere, J. Geophys. Res., 103, 20685-20695, 1998.

Hall, C. M., On the possibility of ion-drag inducing dynamic instability in the lower thermosphere neutral gas, Ann. Geophysicae, 18, 967-971, 2000.

Hedin, A. E., Extension of the MSIS thermosphere model into the middle and lower atmosphere, J. Geophys. Res., 96, 1159-1172, 1991.

Ratcliffe, J.A., An introduction to the ionosphere and magnetosphere, 256 pp., Cambridge University Press, Great Britain, 1972.

Tennekes, H. and Lumley, J. L., A first course in turbulence, 300 pp., MIT, USA, 1972. 